\title{
Navigated Transcranial Magnetic Stimulation
}

National Cancer Institute

\section{Source}

National Cancer Institute. Navigated Transcranial Magnetic Stimulation. NCI Thesaurus. Code $C 162608$.

Transcranial magnetic stimulation that uses image-based knowledge of individual patient brain anatomy for determining the dose and location targeting of stimulation. Navigation combines data on anatomical structures with known delivery of stimulation, forming the basis for dose determination and targeting. 